\title{
ON SUBGRADIENT DUALITY WITH STRONG AND WEAK CONVEX FUNCTIONS
}

\author{
V. JEYAKUMAR \\ (Received 4 April 1984, revised 11 July 1984) \\ Communicated by B. Mond
}

\begin{abstract}
A duality theorem of Wolfe for nonlinear differentiable programs is extended to nondifferentiable programs with strong and weak convex functions, by replacing gradients by local subgradient. A converse duality theorem is also proved.
\end{abstract}

1980 Mathematics subject classification (Amer. Math. Soc.): 90 C 25, 90 C 30, 90 C 48.

\section{Introduction and preliminaries}

Consider the following pair of problems.

(P) Minimize $f_{0}(x)$ subject to $f_{i}(x) \leqslant 0, i=1,2, \ldots, m, x \in C$.

(D) Maximize $f_{0}(\xi)+\sum_{i=1}^{m} \lambda_{i} f_{i}(\xi)$ subject to $\lambda_{i} \geqslant 0, i=1,2, \ldots, m, \xi \in C$, $0 \in \partial^{*} f_{0}(\xi)+\sum_{i=1}^{m} \lambda_{i} \partial^{*} f_{i}(\xi)-(C-\xi)^{*}$.

Here the functions $f_{i}, i=0,1,2, \ldots, m$, are $\rho_{i}$-convex [10] and finitely directionally differentiable on a pre-Hilbert space $X, C$ is a convex subset of $X$, and $\partial^{*} f_{i}(a)$ is the local subdifferential of $f_{i}$ at $a$ in the sense of Craven and Mond [4]. If $f_{i}(i=0,1, \ldots, m)$ are continuous convex functions then this is just the pair of problems for which Schechter [9] proved duality theorems. If $X$ is finite dimensional and the functions $f_{i}, i=0,1,2, \ldots, m$, are differentiable and convex and $C=X$ then the duality between (P) and (D) was proved by Wolfe [12] and converse duality was proved by Craven and Mond [5]. In this paper, we prove these duality results between (P) and (D) with strong and weak convex functions $[10,11]$.

(c) 1986 Australian Mathematical Society $0263-6115 / 86 \$ A 2.00+0.00$ 
The outline of the paper is as follows. In Section 2, necessary optimality conditions of Fritz John and Kuhn Tucker types are derived for a cone-constraints program $\left(\mathrm{P}_{N}\right)$, generalizing the program $(\mathrm{P})$. Here objective and constraint functions are nearly convex [4] (see also the definition below). Then the optimality conditions for (P) are deduced. In Section 3, the duality results for the programs (P) and (D) are obtained by showing that $\rho$-convex functions are nearly convex. Moreover, a converse duality theorem is proved between (P) and (D) in the case $C=X$.

Throughout this paper, $X$ shall denote a (real) pre-Hilbert space with the inner product $\langle$,$\rangle , and norm \|\cdot\|=\langle\cdot, \cdot\rangle^{1 / 2}$, and $Y$ denotes a real normed linear space. Denote by $X^{\prime}$ and $Y^{\prime}$ the (topological) dual spaces of $X$ and $Y$, each with its weak* topology. Let $K \subset X$; then Int $K$ and $\bar{K}$ denote the interior and closure of $K$ respectively. Denote $K^{*}=\left\{v \in X^{\prime}:(\forall k \in K), v(k) \geqslant 0\right\}$, the dual cone of $K$, and Cone $K=\{\lambda k: k \in K, \lambda \geqslant 0\}$, the cone generated by $K$.

If $K$ is a convex cone then $K^{* *}=\overleftarrow{K}$. Let $Y$ be a real normed linear space and $S \subset Y$ a closed convex cone; then a function $f: X \rightarrow Y$ is said to be $S$-convex if

$$
(\forall x, y \in X)(\forall \lambda \in[0,1]), \quad \lambda f(x)+(1-\lambda) f(y)-f(\lambda x+(1-\lambda) y) \in S \text {. }
$$

If $Y=\mathbb{R}$ and $S=\mathbb{R}_{+}$then this reduces to the usual definitions of convex [2]. A real valued function $g: X \rightarrow \mathbb{R}$ is said to be $\rho$-convex [10] if there exists some real number $\rho$ such that for each $x, y \in X$ and $0 \leqslant \lambda \leqslant 1$,

$$
g(\lambda x+(1-\lambda) y) \leqslant \lambda g(x)+(1-\lambda) g(y)-\rho \lambda(1-\lambda)\|x-y\|^{2} .
$$

If $\rho>0$, then the function $g$ is said to be strongly convex. If $\rho=0$, then the function $g$ is convex. If $\rho<0$, then the function $g$ is said to be weakly convex.

We shall make use of the following result of Vial [10], which was proved using the identity

$$
\|x\|^{2}=\|y\|^{2}+2\langle x-y, y\rangle+\|x-y\|^{2} .
$$

LEMmA 1.1. The function $g: X \rightarrow \mathbb{R}$ is $\rho$-convex if and only if there exists a convex function $h: X \rightarrow \mathbb{R}$ such that $g(x)=h(x)+\rho\|x\|^{2}$.

A function $f: X \rightarrow Y$ is said to be weakly directionally differentiable at $a \in X$, if the limit

$$
f^{\prime}(a, x)=\lim _{\lambda \downarrow 0} \lambda^{-1}[f(a+\lambda x)-f(a)]
$$

exists (in the weak topology of $Y$ ), possibly with infinite values for some $x \in X ; f$ is said to be finitely directionally differentiable (f.d.d), if also $f^{\prime}(a, x)$ is finite for every $x \in X$ and bounded above in a neighborhood of some $x$. A directionally differentiable function $f: X \rightarrow Y$ will be called $S$-nearly convex at $a \in X$ if $f^{\prime}(a, \cdot)$ 
is $S$-convex, positively homogeneous and bounded above on a neighborood. If $Y=\mathbb{R}$ and $S=\mathbb{R}_{+}$then this reduces to the equivalent definition of nearly convex at $a$ in the sense of Craven and Mond [4]; (cf. [9]).

Note that the sum of an $S$-convex function and a function which possesses linear Gateaux derivative at $a$ is an $S$-nearly convex function at $a$. Let $g: X \rightarrow \mathbb{R}$ be finitely directionally differentiable at $a \in X$. Then a vector $v \in X^{\prime}$ will be called a local subgradient of $g$ at $a \in X[4,3]$, if, for all $x \in X$,

$$
g(a+\lambda x)-g(a) \geqslant \lambda v(x)+o(\lambda), \quad(\lambda \downarrow 0) .
$$

The local subdifferential $\partial^{*} g(a)$ of $g$ at $a$ is the set of all local subgradients of $g$ at $a$. If $g: X \rightarrow \mathbb{R}$ is nearly convex at a [4, Theorem 2], then $\partial^{*} g(a)$ is a convex weak * compact subset of $X^{\prime}$, and

$$
\partial^{*} g(a)=\bigcap_{x \in X}\left\{v \in X^{\prime}: g^{\prime}(a, x) \geqslant v x\right\} .
$$

We also note that if $\tau$ is nonnegative real, then it follows easily that $\partial^{*}(\tau g)(a)=$ $\tau \partial^{*} g(a)$. If $g$ is a continuous convex function then $\partial^{*} g(a)=\partial g(a)$, the subgradient of $g$ (see Rockafellar [7]).

LEMMA 1.2. If $g_{1}$ and $g_{2}$ are nearly convex at a, then $\partial^{*}\left(g_{1}+g_{2}\right)(a) \subset \partial^{*} g_{1}(a)$ $+\partial^{*} g_{2}(a)$.

Proof. For $h=g_{1}, g_{2}$ and $g_{1}+g_{2}$, [4, Theorem 2] shows that $\partial^{*} h(a)$ is convex and weak * compact, and satisfies $h^{\prime}(a, z)=\max _{\omega \in \partial^{*} h(a)}\langle\omega, z\rangle$. Suppose now that some element $\xi$ of $\partial^{*}\left(g_{1}+g_{2}\right)(a)$ fails to belong to $\partial^{*} g_{1}(a)+\partial^{*} g_{2}(a)$. Then, by the separation theorem [6], there exists $z \in X$ such that

$$
\begin{aligned}
\langle\xi, z\rangle & >\max \left\{\langle\omega, z\rangle: \omega \in \partial^{*} g_{1}(a)+\partial^{*} g_{2}(a)\right\} \\
& =\max \left\{\left\langle\omega_{1}, z\right\rangle: \omega_{1} \in \partial^{*} g_{1}(a)\right\}+\max \left\{\left\langle\omega_{2}, z\right\rangle: \omega_{2} \in \partial^{*} g_{2}(a)\right\} \\
& =g_{1}^{\prime}(a, z)+g_{2}^{\prime}(a, z) .
\end{aligned}
$$

Since $\xi \in \partial^{*}\left(g_{1}+g_{2}\right)(a), \quad\left(g_{1}+g_{2}\right)^{\prime}(a, z) \geqslant\langle\xi, z\rangle>g_{1}^{\prime}(a, z)+g_{2}^{\prime}(a, z)$, a contradiction.

\section{Optimality conditions}

We begin by considering the following nearly convex program $\left(\mathrm{P}_{\mathrm{N}}\right) \quad$ minimize $f(x)$ subject to $-g(x) \in S, \quad x \in C$, in which $X$ is a pre-Hilbert space and $Y$ is a normed linear space; $S \subset Y$ is a closed convex cone with nonempty interior, $C$ is a convex set and $f: X \rightarrow \mathbb{R}$ and $g: X \rightarrow Y$ are nearly convex and $S$-nearly convex at each point of $X$ respectively. 
Craven and Mond [4] established necessary optimality conditions in a slightly complicated form for $\left(\mathrm{P}_{\mathrm{N}}\right)$ in the special case $C=X$, under some strengthened directional differentiability assumptions (namely, arcwise directional differentiability). However, they have not assumed the convex cone $S$ to have nonempty interior. Here we obtain optimality conditions of Fritz John and Kuhn Tucker types for $\left(P_{N}\right)$. These conditions are expressed in terms of local subdifferential.

THEOREM 2.1. Consider the problem $\left(\mathrm{P}_{\mathrm{N}}\right)$; let a be optimal for $\left(\mathrm{P}_{\mathrm{N}}\right)$, and let $f: X \rightarrow \mathbb{R}$ be nearly convex at $a$ and $g: X \rightarrow Y$ an $S$-nearly convex function at $a$. Then there exist $\tau \in \mathbb{R}_{+}$and $\lambda \in S^{*}$, not both zero such that

$$
0 \in \tau \partial^{*} f(a)+\partial^{*}(\lambda g)(a)-(C-a)^{*}, \quad \lambda g(a)=0 .
$$

Proof. Assume that $a$ is a local minimum for $\left(\mathrm{P}_{N}\right)$. Suppose that the system

$$
f^{\prime}\left(a, x_{0}\right)<0, \quad-g^{\prime}\left(a, x_{0}\right)-g(a) \in \operatorname{Int} S
$$

has a solution $x_{0} \in \operatorname{Cone}(C-a)$. Then there exists $\bar{\alpha}>0$ such that $a+\alpha x_{0} \in C$, for each $0<\alpha \leqslant \bar{\alpha}$ and

$$
\begin{aligned}
g\left(a+\alpha x_{0}\right) & =g(a)+g^{\prime}\left(a, x_{0}\right) \alpha+o(\alpha) \\
& =\alpha\left(g(a)+g^{\prime}\left(a, x_{0}\right)\right)+(1-\alpha) g(a)+o(\alpha) \in-S
\end{aligned}
$$

for sufficiently small $\alpha>0$, since $-g(a) \in S$ and $-g^{\prime}\left(a, x_{0}\right)-g(a) \in$ Int $S$. Thus $a+\alpha x_{0}$ is a feasible solution to the problem $\left(\mathrm{P}_{\mathrm{N}}\right)$, for sufficiently small positive $\alpha$. Since $f$ is directionally differentiable at $a, f\left(a+\alpha x_{0}\right)-f(a)=$ $\alpha f^{\prime}\left(a, x_{0}\right)+o(\alpha)<0$, whenever $\alpha$ is sufficiently small positive. This contradicts the local minimum at $a$. It follows that (3) has no solution $x_{0} \in \operatorname{Cone}(C-a)$.

Let $F(x)=\left(f^{\prime}(a, x), g^{\prime}(a, x)+g(a)\right)$. Then the system $x \in \operatorname{Cone}(C-a)$, $-F(x) \in \operatorname{Int}\left(\mathbb{R}_{+} \times S\right)$ has no solution. So by the basic alternative theorem 2.5.1 (Craven [2]), there exist $\tau \in \mathbb{R}_{+}$and $\lambda \in S^{*}$ not both zero such that for all $x \in \operatorname{Cone}(C-a), \tau f^{\prime}(a, x)+\lambda g^{\prime}(a, x)+\lambda g(a) \geqslant 0$, since by the hypothesis, $F$ is $\left(\mathbb{R}_{+} \times S\right)$-convex and Cone $(C-a)$ is convex. Since $0 \in \operatorname{Cone}(C-a)$ and $-g(a) \in S$, for all $x \in \operatorname{Cone}(C-a),(\tau f+\lambda g)^{\prime}(a, x) \geqslant 0$ and $\lambda g(a)=0$. The result will follow, if we show that

$$
\partial^{*}(\tau f+\lambda g)(a) \cap\left\{(C-a)^{*}\right\} \neq \varnothing .
$$

Suppose that $\partial^{*}(\tau f+\lambda g)(a) \cap\left\{(C-a)^{*}\right\}=\varnothing$. Then $\partial^{*}(\tau f+\lambda g)(a) \cap$ $\left\{[\operatorname{Cone}(C-a)]^{*}\right\}=\varnothing$. Since $\partial^{*}(\tau f+\lambda g)(a)$ is a convex weak * compact set, it follows by the separation theorem (Holmes [6]) there exists $\bar{x} \in X$ such that

(4) $\operatorname{Sup}\left\{v(\bar{x}): v \in \partial^{*}(\tau f+\lambda g)(a)\right\}<\operatorname{Int}\left\{w(\bar{x}): w \in[\operatorname{Cone}(C-a)]^{*}\right\}$.

Since $[\operatorname{Cone}(C-a)]^{*}$ is a cone, the right hand side of (4) must be either 0 or $-\infty$. Also, because the left hand side of (4) is finite, this has two implications: $(\tau f+\lambda g)^{\prime}(a, \bar{x})<0$ and $\bar{x} \in[\operatorname{Cone}(C-a)]^{* *}=\overline{\operatorname{Cone}}(C-a)$. Since 
$(\tau f+\lambda g)^{\prime}(a, \cdot)$ is bounded above in some neighbourhood and convex, by the theorem of Holmes [6, page 82] $(\tau f+\lambda g)^{\prime}(a, \cdot)$ is continuous. So $(\tau f+\lambda g)^{\prime}(a, x)<0$, for all $x$ in some neighbourhood of $\bar{x}$ and in particular for some point $x \in \operatorname{Cone}(C-a)$. This is a contradiction.

These Fritz John type conditions (2), will lead to corresponding Kuhn Tucker type conditions, under any constraint qualification which gives $\tau \neq 0$. Consider the following constraint qualifications:

$$
\begin{gathered}
g^{\prime}(a, \text { Cone }(C-a))+\{\gamma g(a): \gamma \geqslant 0\}+S=Y, \\
-g^{\prime}(a, \text { Cone }(C-a)) \cap \text { Int } S \neq \varnothing .
\end{gathered}
$$

Note that (5) is a directional derivative version of Zowe and Kurcyusz [13] constraint qualification.

THEOREM 2.2. In the problem $\left(\mathrm{P}_{\mathrm{N}}\right)$, let the Fritz John type conditions (2) hold at the feasible point $a$ and let either of the constraint qualifications (5) and (6) hold there. Then $\tau \neq 0$, so that (2) holds with $\tau=1$.

Proof. Suppose that $\tau=0$; then $0 \neq \lambda \in S^{*}$ and (2) gives that

$$
0 \in \partial^{*}(\lambda g)(a)-(C-a)^{*} \Rightarrow \lambda g^{\prime}(a, \text { Cone }(C-a)) \geqslant 0 \text {. }
$$

Since $0 \neq \lambda$ and linear, there exists $y_{0} \in Y$ such that $\lambda\left(y_{0}\right)<0$. If (5) holds, then there exist $x_{0} \in \operatorname{Cone}(C-a)$ and $\gamma_{0} \geqslant 0$ and $s_{0} \in S$ such that $0>\lambda\left(y_{0}\right)=$ $\lambda g^{\prime}\left(a, x_{0}\right)+\lambda\left(\gamma_{0} g(a)\right)+\lambda\left(s_{0}\right) \geqslant 0$. This is a contradiction. If (6) holds then the system

$$
x \in \operatorname{Cone}(C-a), \quad-g^{\prime}(a, x) \in \operatorname{Int} S
$$

has a solution and (7) holds. This contradicts the basic alternative Theorem 2.5.1 [2].

Corollary 2.1. Consider the problem (P) in which $f_{i}, i=0,1,2, \ldots, m$, are nearly convex at a, where $a$ is a local minimum for $(\mathrm{P})$. Let either of the constraint qualifications (5) and (6) with $g=\left(f_{i}\right)_{i=1}^{m}, S=\mathbb{R}_{+}^{m}$ and $Y=\mathbb{R}^{m}$ be satisfied. Then there exist Lagrange multipliers $\lambda_{i} \geqslant 0$, for $i=1,2, \ldots, m$, not all zero such that (8)

$$
0 \in \partial^{*} f_{0}(a)+\sum_{i=1}^{m} \lambda_{i} \partial^{*} f_{i}(a)-(C-a)^{*}, \quad \lambda_{i} f_{i}(a)=0, \quad i=1,2, \ldots, m .
$$

Proof. The result follows immediately from Theorem 2.2 by taking $f_{0}=f$ and $g(x)=\left(f_{i}(x)\right)_{i=1}^{m}$.

Remark 2.1. If we assume that $X$ is complete, $f_{0}$ satisfies a locally Lipschitz condition at each point, $f_{0}^{\prime}(a, \cdot)$ is lower semicontinuous and $f_{i}, i=1,2, \ldots, m$, are continuous convex functions, then Corollary $2.1 \mathrm{can}$ be obtained as a special 
case of Schechter [9, Theorem 1.2]. Further, if we add the hypothesis that $C$ is also bounded, then we would have a result similar to Clarke [1, Proposition 13]. However, for nonconvex functions, $\partial^{*} f(a)$ does not necessarily coincide with Clarke's $\partial_{c} f(a)$.

\section{The duality results}

In this section we obtain a duality relationship between (P) and (D) using the results of the last section. First we show that $\rho$-convex functions are nearly convex. Throughout Sections 3 and 4 we shall consider finitely directionally differentiable $\rho$-convex functions.

LEMMA 3.1. $\rho$-convex functions are nearly convex at each point where the functional value is finite.

Proof. The proof immediately follows by noting that a $\rho$-convex function is a sum of a convex function and a differentiable function (see Lemma 1.1), which is nearly convex.

Lemma 3.2. Let $f: X \rightarrow \mathbb{R}$ be directionally differentiable on $X$. If $f$ is $\rho$-convex then for all $x, a \in X, f(x)-f(a) \geqslant f^{\prime}(a, x-a)+\rho\|x-a\|^{2}$.

Proof. Since $f$ is $\rho$-convex, there exists a convex function $h: X \rightarrow \mathbb{R}$ such that $f(x)=h(x)+\rho\|x\|^{2}$. Thus

$$
(\forall x, a \in X) \quad h(x)-h(a) \geqslant h^{\prime}(a, x-a) .
$$

From the identity (1), we get

$$
\rho\|x\|^{2}-\rho\|a\|^{2}=2 \rho\langle a, x-a\rangle+\rho\|x-a\|^{2} .
$$

Then, by adding (9) and (10) we have the result.

Theorem 3.1 (Weak Duality). Let $x$ be feasible for $(\mathrm{P})$ and $(\xi, \lambda)$ feasible for (D). If $f_{i}, i=0,1,2, \ldots, m$, are $\rho_{i}$-convex and if $\left(\rho_{0}+\sum_{i=1}^{m} \lambda_{i} \rho_{i}\right) \geqslant 0$ then

$$
f_{0}(x) \geqslant f_{0}(\xi)+\sum_{i=1}^{m} \lambda_{i} f_{i}(\xi)
$$

Proof. Since $(\xi, \lambda)$ is feasible for (D), there exist $u \in \partial^{*} f_{0}(\xi)$ and $v_{i} \in \partial^{*} f_{i}(\xi)$ such that

$$
\left(u+\sum_{i=1}^{m} \lambda_{i} v_{i}\right)(x-\xi) \geqslant 0, \quad \forall x \in C .
$$


Thus,

$$
\begin{aligned}
& f_{0}(x)-\left[f_{0}(\xi)+\sum_{i=1}^{m} \lambda_{i} f_{i}(\xi)\right] \\
& \geqslant f_{0}^{\prime}(\xi, x-\xi)+\rho_{0}\|x-\xi\|^{2}-\sum_{i=1}^{m} \lambda_{i} f_{i}(\xi) \quad \text { (by Lemma 3.2) } \\
& \geqslant u(x-\xi)-\sum_{i=1}^{m} \lambda_{i} f_{i}(\xi)+\rho_{0}\|x-\xi\|^{2} \quad\left(\text { since } u \in \partial^{*} f_{0}(\xi)\right) \text {. } \\
& \geqslant-\sum^{m} \lambda_{i} v_{i}(x-\xi)-\sum_{i=1}^{m} \lambda_{i} f_{i}(\xi)+\rho_{0}\|x-\xi\|^{2} \quad(\text { by }(11)) \\
& \geqslant-\sum^{m} \lambda_{i} f_{i}^{\prime}(\xi, x-\xi)-\sum \lambda_{i} f_{i}(\xi)+\rho_{0}\|x-\xi\|^{2} \quad\left(\text { since } v_{i} \in \partial^{*} f_{i}(\xi)\right) \\
& \geqslant-\sum_{i=1}^{m} \lambda_{i} f_{i}(x)+\sum_{i=1}^{m} \lambda_{i} \rho_{i}\|x-\xi\|^{2}+\rho_{0}\|x-\xi\|^{2} \quad \text { (by Lemma (3.2)) } \\
& =-\sum_{i=1}^{m} \lambda_{i} f_{i}(x)+\left(\rho_{0}+\sum_{i=1}^{m} \lambda_{i} \rho_{i}\right)\|x-\xi\|^{2}, \\
& \geqslant-\sum_{i=1}^{m} \lambda_{i} f_{i}(x) \quad \text { (by the hypothesis) } \\
& \geqslant 0 \quad(\text { since } x \text { is feasible for }(P))
\end{aligned}
$$

THEOREM 3.2 (Strong Duality). Suppose that a is optimal for (P) and the Kuhn Tucker conditions (8) hold at the point a. If $\left(\rho_{0}+\sum_{i=1}^{m} \bar{\lambda}_{i} \rho_{i}\right) \geqslant 0$ for each feasible $(\bar{\xi}, \bar{\lambda})$ of $(\mathrm{D})$, then $(a, \lambda)$ is optimal for (D), and the optimal values of (P) and (D) are equal.

Proof. Since Kuhn Tucker conditions (8) hold at the optimal point $a$, we have $0 \in \partial^{*} f_{0}(a)+\sum_{i=1}^{m} \lambda_{i} \partial^{*} f_{i}(a)-(C-a)^{*}$ and $\lambda_{i} f_{i}(a)=0, i=1,2, \ldots, m$. Therefore, $(a, \lambda)$ is feasible for (D). Moreover, since $\lambda_{i} f_{i}(a)=0$, for $i=1,2, \ldots, m$, we have $\operatorname{Max}(\mathrm{D}) \geqslant f(a)+\sum_{i=1}^{m} \lambda_{i} f_{i}(a)=f(a) \geqslant \operatorname{Min}(\mathrm{P})$. This with weak duality, shows that $\operatorname{Max}(\mathrm{D})=\operatorname{Min}(\mathrm{P})$.

REMARK 1. The following geometric interpretations could be given to ensure the duality between (P) and (D). If, in the problem (P), the constraint functions $f_{i}$, $i=1,2, \ldots, m$, are weakly convex (not convex) then the objective function should be strongly convex. Conversely, if the objective function is weakly convex, then at least one of the constraint functions should be strongly convex. 
REMARK 2. If all functions $f_{i}, i=0,1,2, \ldots, m$, involved in the program (P) are convex (i.e. $\rho_{i}=0, i=0,1,2, \ldots, m$ ) then our results reduce to Theorems 2.1 and 2.2 of Schechter [9]. So, this in turn extended results of Wolfe [12] and Schechter $[8,9]$ to non-convex programs.

\section{Converse duality}

Here we are interested in proving a converse duality theorem, using the Craven and Mond [4] approach in a simplified manner, for the problem $(P)$ in the case $C=X$ :

$\left(\mathrm{P}^{\prime}\right)$ minimize $f_{0}(x)$ subject to $f_{i}(x) \leqslant 0, i=1,2, \ldots, m$.

First we consider the statement

$$
0 \in \partial^{*} f_{0}(\xi)+\sum_{i=1}^{m} \partial^{*}\left(\lambda_{i} f_{i}\right)(\xi)
$$

Assume that the statement (Q) is satisfied by $\xi=a$ and $\lambda=\bar{\lambda} \in \mathbb{R}_{+}^{m}$. The statement $(\mathrm{Q})$ is said to be "solvable near $a$ " if, for some $\delta>0$, whenever $\|\lambda-\bar{\lambda}\|<\delta$, the statement (Q) possesses a solution $(\xi, \lambda)$ for which $\|\xi-a\| \rightarrow 0$ as $\|\lambda-\bar{\lambda}\| \rightarrow 0$.

Note that, if $f_{i}, i=0,1,2, \ldots, m$, are continuously Fréchet differentiable at $a$, then a sufficient condition for the statement (Q) to be solvable near $a$ is that $f_{0}^{\prime \prime}(a)+(\bar{\lambda} f)^{\prime \prime}(a)$ is bijective, where $f=\left(f_{i}\right)_{i=1}^{m}$ (for details see Craven [2, page 73] and [4]).

Now, we shall prove the general converse duality theorem.

Consider the following dual problem to $\left(\mathrm{P}^{\prime}\right)$.

(D') Maximize $f_{0}(\xi)+\sum_{i=1}^{m} \lambda_{i} f_{i}(\xi)$ subject to $\lambda_{i} \geqslant 0,0 \in \partial^{*} f_{0}(\xi)+$ $\sum_{i=1}^{m} \partial^{*}\left(\lambda_{i} f_{i}\right)(\xi)$

TheOREM 4.1 (Converse Duality Theorem). For the problem $\left(\mathrm{P}^{\prime}\right)$, let $f_{i}: X \rightarrow \mathbb{R}$, $i=0,1,2, \ldots, m$, be continuous and $\rho_{i}$-convex functions. Assume that $\left(\mathrm{D}^{\prime}\right)$ attains $a$ maximum at $\xi=a, \lambda=\bar{\lambda}$. If the statement $(\mathrm{Q})$ is solvable near $a$, then the problem $\left(\mathrm{P}^{\prime}\right)$ is dual to $\left(\mathrm{D}^{\prime}\right)$ provided that $\left(\rho_{0}+\sum_{i=1}^{m} \bar{\lambda}_{i} \rho_{i}\right) \geqslant 0$.

Proof. As we have proved the weak duality theorem, it is enough if we prove $f_{i}(a) \leqslant 0, i=1,2, \ldots, m$, and $\sum_{i=1}^{m} \bar{\lambda}_{i} f_{i}(a)=0$. Now, by hypothesis, there exist $u \in \partial f_{0}(a)$ and $v_{i} \in \partial^{*}\left(\bar{\lambda}_{i} f_{i}\right)(a), i=1,2, \ldots, m$, such that $0=u+\sum_{i=1}^{m} v_{i}$. Let $t \in \mathbb{R}^{m}$. Since (Q) is solvable near $a$, whenever $\lambda=\bar{\lambda}+\alpha t \in \mathbb{R}_{+}^{m}$ and $\alpha$ is sufficiently small positive, (Q) has a solution $(a+\xi(\alpha t), \bar{\lambda}+\alpha t)$ where $\xi(\alpha t) \rightarrow 0$ 
as $\alpha \downarrow 0$. Therefore, $(a+\xi(\alpha t), \bar{\lambda}+\alpha t)$ satisfies the constraint of $\left(\mathrm{D}^{\prime}\right)$. Since $\left(\mathrm{D}^{\prime}\right)$ is maximized at $(a, \bar{\lambda})$,

$$
\begin{aligned}
0 \geqslant & f_{0}(a+\xi(\alpha t))+\sum_{i=1}^{m}\left(\bar{\lambda}_{i}+\alpha t_{i}\right) f_{i}(a+\xi(\alpha t))-\left[f_{0}(a)+\sum_{i=1}^{m} \bar{\lambda}_{i} f_{i}(a)\right] \\
\geqslant & \langle u, \xi(\alpha t)\rangle+\rho_{0}\|\xi(\alpha t)\|^{2}+\sum_{i=1}^{m}\left\langle v_{i}, \xi(\alpha t)\right\rangle \\
& +\bar{\lambda}_{i} \rho_{i}\|\xi(\alpha t)\|^{2}+\sum_{i=1}^{m} \alpha t_{i} f_{i}(a+\xi(\alpha t))
\end{aligned}
$$

(by Lemma 3.2 and the definition of the local subdifferential)

$$
\begin{aligned}
& =\left\langle u+\sum_{i=1}^{m} v_{i}, \xi(\alpha t)\right\rangle+\alpha \sum_{i=1}^{m} t_{i} f_{i}(a+\xi(\alpha t))+\left(\rho_{0}+\sum_{i=1}^{m} \bar{\lambda}_{i} \rho_{i}\right)\|\xi(\alpha t)\|^{2} \\
& =0+\alpha \sum_{i=1}^{m} t_{i} f_{i}(a+\xi(\alpha t))+\left(\rho_{0}+\sum_{i=1}^{m} \bar{\lambda}_{i} \rho_{i}\right)\|\xi(\alpha t)\|^{2} \\
& \geqslant \alpha \sum_{i=1}^{m} t_{i} f_{i}(a+\xi(\alpha t)) \quad\left(\text { since }\left(\rho_{0}+\sum_{i}^{m} \bar{\lambda}_{i} \rho_{i}\right) \geqslant 0\right) .
\end{aligned}
$$

Now, by letting $\alpha \downarrow 0$, we get $0 \geqslant \sum_{i=1}^{m} t_{i} f_{i}(a)=\langle t, f(a)\rangle$ (since $f_{i}$ is continuous and $\left.f=\left(f_{i}\right)_{i=1}^{m}\right)$. As this is true for all $t \in \mathbb{R}^{m}, f_{i}(a) \leqslant 0, i=1,2, \ldots, m$. Also, by taking $t=-\bar{\lambda} / 2$, we get $\langle\bar{\lambda}, f(a)\rangle \geqslant 0$. Hence $\sum_{i=1}^{m} \bar{\lambda}_{i} f_{i}(a)=0$.

\section{Acknowledgement}

I would like to thank my supervisor Dr. B. D. Craven for his valuable suggestions and helpful guidance, without which this paper may not have been completed. The author is grateful to the unknown referee for helpful comments and suggestions towards the improvement of this paper.

\section{References}

[1] F. H. Clarke, 'A new approach to Lagrange multipliers,' Math. Oper. Res. 1 (1976), 165-174.

[2] B. D. Craven, Mathematical programming and control theory, (Chapman and Hall, London, 1978).

[3] B. D. Craven, 'Non-differentiable Pontryagin principle,' Research Report No. 20, (1977), Department of Mathematics, University of Melbourne. 
[4] B. D. Craven and B. Mond, 'Lagrange conditions for quasidifferentiable optimization', pp. 177-191, IX Intern. Symp. on Math. Prog. (Budapest, in Surveys of Mathematical Programming, Vol. 1, A. Prekopa (ed.), North-Holland, Amsterdam, (1976)).

[5] B. D. Craven and B. Mond, 'On converse duality in nonlinear programming,' Operations Res. 19 (1971), 1075-1078.

[6] R. B. Holmes, Geometric functional analysis and its applications (Springer-Verlag, 1975).

[7] R. T. Rockafellar, Convex analysis (Princeton University Press, Princeton, 1972).

[8] M. Schechter, 'A subgradient duality theorem', J. Math. Anal. Appl. 61 (1977), 850-855.

[9] M. Schechter, 'More on subgradient duality', J. Math. Anal. Appl. 71 (1979), 251-262.

[10] J. P. Vial, 'Strong and weak convexity of sets and functions', Math. Oper. Res. 8 (1983), 231-259.

[11] J. P. Vial, 'Strong convexity of sets and functions', J. Math. Econom. 9(1982), 187-205.

[12] P. Wolfe, 'A duality theorem for nonlinear programming', Quart. Appl. Math. 19 (1961), 239-244.

[13] J. Zowe and S. Kureyusz, 'Regularity and stability for the mathematical programming problems in Banach spaces', Appl. Math. Optim. 5 (1979), 49-62.

\section{Department of Mathematics}

University of Melbourne

Parkville, Victoria 3052

Australia 